\title{
Building efficient fuzzy regression trees for large scale and high dimensional problems
}

\author{
Javier Cózar ${ }^{1 *}$ (D) Francesco Marcelloni², José A. Gámez ${ }^{1}$ and Luis de la Ossa ${ }^{1}$
}

*Correspondence: javier.cozar@uclm.es ${ }^{1}$ Departamento de Sistemas Informáticos/I3A, Universidad de Castilla-La Mancha, 02071 Albacete, Spain Full list of author information is available at the end of the article

\begin{abstract}
Regression trees (RTs) are simple, but powerful models, which have been widely used in the last decades in different scopes. Fuzzy RTs (FRTs) add fuzziness to RTs with the aim of dealing with uncertain environments. Most of the FRT learning approaches proposed in the literature aim to improve the accuracy, measured in terms of mean squared error, and often neglect to consider the computation time and/or the memory requirements. In today's application domains, which require the management of huge amounts of data, this carelessness can strongly limit their use. In this paper, we propose a distributed FRT (DFRT) learning scheme for generating binary RTs from big datasets, that is based on the MapReduce paradigm. We have designed and implemented the scheme on the Apache Spark framework. We have used eight real-world and four synthetic datasets for evaluating its performance, in terms of mean squared error, computation time and scalability. As a baseline, we have compared the results with the distributed RT (DRT) and the Distributed Random Forest (DRF) available in the Spark MLlib library. Results show that our DFRT scales similarly to DRT and better than DRF. Regarding the performance, DFRT generalizes much better than DRT and similarly to DRF.
\end{abstract}

Keywords: Fuzzy regression trees, Big Data, Fuzzy discretizer, Apache Spark

\section{Introduction}

Regression trees (RTs) are light but powerful models that have been extensively used in different application domains [13, 24, 35, 37]. Their main advantage relies on their simplicity both in the learning and execution phases. Furthermore, RTs are highly interpretable models, that is, they can be used to describe the relation between the inputs and the output. An RT is a tree-shaped directed graph in which each internal (non-leaf) node represents a test on an attribute and each leaf node holds an output value. Each branch, which consists of a sequence of non-leaf nodes (tests) and a leaf node (output value), represents a possible outcome for such group of tests. By following the corresponding branches from the root node to a leaf, one can find an explanation of how the output has been determined. In the literature, several different algorithms for learning RTs have been proposed [5, 20,36].

The type of tests used in the internal nodes determines the partitioning of the input space. Given a parent node, the test acts as a splitting method for generating its child nodes. There are two main categories of RTs: binary and multi-way RTs. Binary RTs

(c) The Author(s) 2018. This article is distributed under the terms of the Creative Commons Attribution 4.0 International License (http://creativecommons.org/licenses/by/4.0/), which permits unrestricted use, distribution, and reproduction in any medium, provided you give appropriate credit to the original author(s) and the source, provide a link to the Creative Commons license, and indicate if changes were made. 
recursively split the input space into two subspaces for each test node, so that each parent node is connected exactly to two child nodes. On the other hand, multi-way RTs split the input space into $k$ partitions, where $k>1$. Therefore, each parent node is connected to a not fixed number of child nodes. The structure of a multi-way RT is usually more compact and interpretable than the structure of a binary RT $[3,22]$. On the other hand, multi-way splits lead to more accurate RTs but, since they tend to fragment the training data very quickly [19], they generally need larger training datasets to work effectively [22]. Furthermore, a multi-way RT can be also represented as a binary RT [14].

One of the main drawbacks of RTs is related to the crisp bounds of the branch conditions: a small change in the values of the input variables may produce an important difference in the prediction. To overcome this problem, RTs have been extended with the use of fuzzy set theory, originating fuzzy regression trees (FRTs) [31, 39]. FRTs employ fuzzy conditions in their internal nodes. Therefore, an input can enable several branches in a test node, and reach different leaves, with different confidence levels. The output value of the FRT is obtained by appropriately combining the values associated with these multiple leaf nodes. FRTs are, in general, more complex both in learning and execution phases than RTs.

Fuzzy regression tree learning algorithms usually apply an initial discretization step aimed at generating fuzzy partitions on the continuous input variables, typically guided by some purposely-defined index $[43,47]$. Since this step has a direct impact on the performance of the learning algorithm, several studies evaluate how the accuracy and complexity of the generated models depend on the discretization $[15,23,50]$.

Most of FRT learning algorithms proposed in the literature focus on the accuracy but do not consider the computation complexity and space requirements [33,39]. Typically, these algorithms have been tested and assessed on small datasets, and are not generally suitable for managing huge amounts of data. In fact, one solution often adopted to work in real contexts with these algorithms, is to reduce the dataset by applying downsampling techniques. However, these techniques may cut off some useful knowledge, making FRT learning approaches purposely designed for managing the overall dataset more desirable and effective. Thus, we aim to learn FRTs from the overall dataset, independently of its size. In other words, we desire to cope explicitly with Big Data.

As described in [42], Big data refers to the storage and analysis of large and/or complex data sets using a series of specific techniques. These techniques combine new paradigms (software) together with specific hardware architectures. A recent work [38] of one of the co-authors has proposed a novel fuzzy decision tree algorithm to address Big Data classification problems. First, the domain of the continuous input features is discretized by adopting a distributed approach based on the fuzzy entropy concept [50]. Then, the fuzzy decision tree is learned by applying a distributed learning algorithm based on the fuzzy information gain concept. Both phases have been implemented by using the MapReduce paradigm in the Apache Spark framework.

In this paper, we adapt the algorithm proposed in [38] to deal with Big Data regression problems. In particular, we adopt more suitable metrics than fuzzy entropy and information gain for the discretization and FRT learning phases. Furthermore, we employ some specific arrangements for speeding-up the distributed implementation of the FRT. Finally, we perform an extensive experimentation of our FRT learning algorithm by using 
eight real-world datasets [2] and four synthetic datasets [40]. The results are discussed in terms of mean squared error, computation time and scalability, and compared with the ones achieved by the distributed regression tree and the Distributed Random Forest [4], both included in the MLlib Apache Spark library [30]. To the best of our knowledge, the proposed algorithm is the first distributed FRT learning scheme proposed in the literature.

The paper is organized as follows. "Background" section introduces some preliminary concepts about RTs and FRTs. Afterwards, in "MapReduce and spark" section, we briefly discuss MapReduce and Apache Spark. "Fuzzy regression tree for Big Data" section describes our proposed FRT learning algorithm and presents its distributed implementation. Then, in "Results and discussion" section, we present the experimental results. Finally, in "Conclusions" section, we draw our conclusions.

\section{Background}

In classification problems, a class $c_{m}$ from a predefined set $\mathcal{C}=\left\{c_{1}, \ldots, c_{M}\right\}$ of $M$ classes is assigned to an unlabeled instance. A classification problem is defined by a set of input variables $\mathbf{X}=\left\{X_{1}, \ldots, X_{F}\right\}$ and the output $Y$. In case of numerical variables, $X_{f}$ is defined on a universe $U_{f} \subset \Re$. In case of categorical variables, $X_{f}$ is defined on a set $L_{f}=\left\{L_{f, 1}, \ldots, L_{f, T_{f}}\right\}$ of categorical values.

Decision trees (DTs) have been widely used in instance classification problems $[11,16$, 52]. A DT is a tree-shaped directed graph in which each internal (non-leaf) node represents a test on an attribute. Each path from the root to a leaf corresponds to a sequence of tests, which aims to isolate a subspace of the input space. The objective of the test is to partition the training set into subsets as pure as possible, that is, subsets composed by instances belonging to the same class. In the case of numerical variables, tests are defined by intervals in the definition domain; in the case of categorical variables, they correspond to subsets of the possible categorical values. Formally, tests are in the form of $X_{f}>x_{f, s}$ and $X_{f} \leq x_{f, s}$ for numerical input variables, and $X_{f} \subseteq \mathbf{L}_{f, s}$ for categorical input variables, where $x_{f, s}$ and $\mathbf{L}_{f, s}$ are a numeric threshold and a set of categorical values for the test $s$, respectively. Each leaf node is characterized by a class $c_{m} \in \mathcal{C}:$ the instances, which satisfy the overall sequence of tests from the root to the leaf, are classified as belonging to $c_{m}$.

Decision trees can also be used in regression problems, named regression trees (RTs). The objective is to predict a real value rather than a class label. Thus, leaf nodes are characterized by a regression model defined over the input variables. For instance M5 [36] generates first-order polynomials using the overall set of input variables. CART [5], which is one of the most known algorithms for generating regression trees, uses zeroorder polynomials i.e. a numerical constant, leading to simpler and usually more robust models. Indeed, the use of high order polynomials tends to overfit.

In this work, we focus on zero-order polynomial fuzzy regression trees (FRTs) [18]. Each real input variable is partitioned by using fuzzy sets. The tests in the internal nodes use these fuzzy sets in the form of $X_{f}$ is $B_{f, t}$, where $B_{f, t}$ is a fuzzy set defined over the variable $X_{f}$. The membership degree of a value $x_{i, f}$ with respect to the fuzzy set $B_{f, t}$ is represented as $\mu_{B_{f, t}}\left(x_{i, f}\right)$. Similarly, the membership degree of an instance $\mathbf{x}_{i}$ with respect to the fuzzy sets $B_{1, t_{1}}, \ldots, B_{F, t_{F}}$ is represented as $T$-Norm $\left(B_{1, t_{1}}\left(\mathbf{x}_{i}\right), \ldots, B_{F, t_{F}}\left(\mathbf{x}_{i}\right)\right)$, which 
is a conjunction of the individual membership degrees. ${ }^{1}$ Since fuzzy sets generally overlap, an input instance may activate more than one leaf node. The value assigned to each leaf node is computed as a weighted average of the output values for all the training set instances that activate such leaf node, using the activation degrees as instance weights.

There are different approaches for generating the fuzzy partitions of the input variables automatically from data $[23,27,50]$. Most of them use two steps: discretization and fuzzy partition generation. The first step consists of discretizing the domain of each input variable into a finite set of disjoint bins. To this aim, different strategies have been proposed in the literature [17]: splitting vs. merging, supervised vs. unsupervised, dynamic vs. static, and global vs. local. Once the bins have been generated, in the second step the membership functions of the fuzzy partition are derived from these bins by employing different methods: the use of the bin bounds to define the fuzzy sets sequentially ordered [34], based on histograms [29], probabilities [12], fuzzy nearest neighbors [21], neural networks [29], entropy [6], particle swarm optimization [32] or clustering based methods [29]. In this paper, we adopt an approach that generates triangular fuzzy partitions directly by using a single step.

Algorithm 1 shows the scheme of a generic FRT learning process, where $\mathbf{T R}=\left\{\left(\mathbf{x}_{1}, y_{1}\right), \ldots,\left(\mathbf{x}_{N}, y_{N}\right)\right\}$ is the training set, $\mathbf{X}$ is the set of input variables, SplitMet is the splitting method, StopMet is the stopping method, and FRT is the output (learnt model).

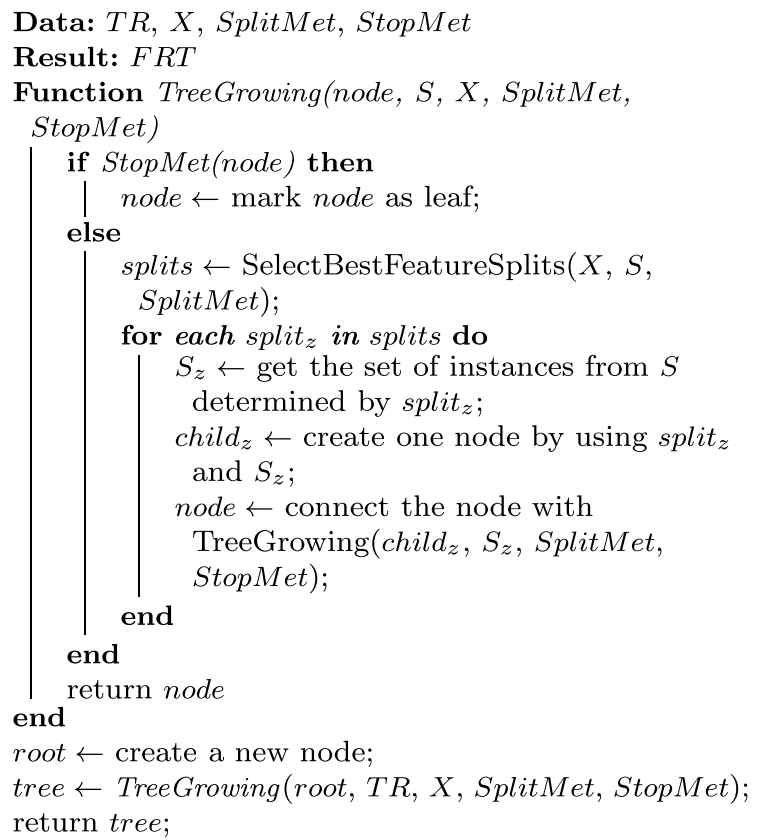

Algorithm 1: Pseudo-code of a generic FRT learning process.

The algorithm first creates the root node. Then, it builds the FRT model with the TreeGrowing recursive function. This function first checks if the node is a leaf by means of the 
StopMet function. If not, it calls the function SelectBestFeatureSplits, which selects the best input variable to partition the input space. There are several metrics that can be used to perform the variable selection. However, the most popular one in zero-order FRTs is the variance $[7,10]$. Finally, for each split $z_{z}$ in splits the TreeGrowing function creates a child node and recursively calls itself using the subset of instances, which satisfy the test for such child node.

After learning the tree structure, each leaf node $n_{i}$ in the set of leaf nodes, $L N$, estimates a regression model from the instances in the training set, which activate such leaf node, i.e., which satisfy the sequence of tests from the root to such leaf node. Let $S_{n_{i}}$ be the set of instances that activate the leaf node $n_{i} . R M_{n_{i}}$ is the weighted average of the instances $\left(x_{l}, y_{l}\right) \in S_{n_{i}}$ by using their activation degree $\mu_{n_{i}^{l}}\left(\mathbf{x}_{l}\right)$ as weight:

$$
R M_{n_{i}}=\frac{\sum_{\left(x_{l}, y_{l}\right) \in S_{n_{i}}} y_{l} \cdot \mu_{n_{i}^{l}}\left(\mathbf{x}_{l}\right)}{\sum_{n_{i} \in L N} \sum_{\left(x_{l}, y_{l}\right) \in S_{n_{i}}} \mu_{n_{i}^{l}}\left(\mathbf{x}_{l}\right)}
$$

During the inference, the prediction is computed as follows. Let $N^{l}=\left\{n_{1}^{l}, \ldots, n_{M^{l}}\right\}$ be the set of leaf nodes activated by the input instance $\mathbf{x}_{l}$ with an activation degree $\mu_{n_{i}^{l}}\left(\mathbf{x}_{l}\right)$. The output of the FRT is obtained as:

$$
\operatorname{FRT}\left(\mathbf{x}_{l}\right)=\frac{\sum_{n_{i} \in \mathbf{N}^{\mathbf{l}}} R M_{n_{i}} \cdot \mu_{n_{i}}\left(\mathbf{x}_{l}\right)}{\sum_{n_{i} \in \mathbf{N}^{\mathbf{l}}} \cdot \mu_{n_{i}}\left(\mathbf{x}_{l}\right)}
$$

\section{MapReduce and Spark}

MapReduce (MR) is a programming paradigm proposed in 2004 [9] for distributing the computation of parallel data-oriented tasks across a cluster of machines. At the beginning, it was developed as a solution to improve the performance of the Google Search Engine. Currently, its usage has been extended to process large amounts of data in multiple different areas [25].

MapReduce presents an abstraction layer to the developers: It divides the problem into smaller tasks, called Map and Reduce, and executes those tasks in parallel taking care of communication, network bandwidth, disk usage and possible failures. In this way, the developers are able to implement parallel algorithms by simply defining the Map and Reduce functions, avoiding problems related to the underlying architecture and hardware configuration. In order to execute these tasks, MR follows a masterslave scheme. First, the data is automatically divided into a set of independent blocks, called chunks, which can be processed in parallel by different machines [26]. Then, the master computing node configures multiple tasks, each one fed with a chunk of data, and executes them in parallel in slave computing nodes.

The data managed by the MR workflow are represented by $\langle k e y, v a l u e\rangle$ pairs. Initially, an arbitrary key is assigned to each input. Then, in the Map phase, each task processes the data by applying the defined map function, which generates a list of intermediate $\langle k e y, v a l u e\rangle$ pairs (one per map task). Then, the system collects these pairs, sort them according to their keys, and feed reduce tasks with the pairs sharing the same key. Finally, in the Reduce phase, the defined reduce function is used to 
combine the list of values (which share the same key) to produce a new $\langle k e y, v a l u e\rangle$ pair. The result of the MapReduce process is a list of $\langle k e y$, value $\rangle$ pairs generated by all the executed Reduce tasks.

The most extended implementation of this paradigm is Apache Hadoop [45], where the open-source community worked together with many private companies to provide a general framework for Big Data processing. The key parts of Apache Hadoop are the MapReduce implementation and the Hadoop Distributed FileSystem (HDFS), which allows working efficiently with large amount of data following the previously described processing.

The most important drawback of Apache Hadoop is that the output is written in the hard driven storage. This decreases the performance for iterative (or stream-based) workflows. Apache Spark [49] is a framework, which overcomes this problem by storing the data structures in the main memory (significantly faster) until the whole process finishes (the process can be a sequence of MapReduce tasks). In practice, the main difference with respect to Apache Hadoop is the resilient distributed dataset (RDD), which relax the MapReduce workflow and allows the developer to define and use additional operations over the data.

Spark has been implemented using the Scala programming language, but there are alternative languages, which can be used for developers, like Python and R. The existence of such interfaces eases its usage and has made Apache Spark one of the most popular frameworks for Big Data processing, in particular within the Data Science community. As a consequence, MLlib [48] has been developed as part of the Apache Spark project. MLlib is a library for Apache Spark, which implements the most popular machine learning algorithms following the MapReduce paradigm.

\section{Fuzzy regression tree for Big Data}

In this section, we present our FRT learning approach for big data regression problems. We have adapted the distributed fuzzy decision tree learning approach, proposed by one of the authors in [38] for handling Big Data classification problems, to deal with regression problems. The learning approach consists of two steps: the first one creates fuzzy partitions on the domains of the input variables, and the second one induces a binary FRT from data using the fuzzy partitions generated in the first step. With respect to the original approach, we have employed different measures for both evaluating the partitions in fuzzy partitioning and for determining the optimal variable to be used in a decision node in the FRT learning phases. Consequently, we have had to introduce different stopping conditions. Further, we have employed some specific adjustments for speeding-up the FRT generation.

In the following, we first describe both steps and then we explain how these steps have been designed to be implemented under the MapReduce paradigm.

\section{Fuzzy partitioning}

Partitioning of continuous variables is a crucial aspect in the generation of both FDTs and FRTs, and therefore should be performed carefully. The work in [50] evaluates several approaches for FDTs. Among them, fuzzy partitioning based on fuzzy entropy proved to be very effective [38]. Most of these metrics are not suitable for fuzzy partitioning in regression problems, which requires specific indexes for measuring the quality of the partition. 
In regression, one of the most used indexes is fuzzy variance [8]. Thus, we have adapted the recursive supervised fuzzy partitioning method proposed in [38] for classification problems, by using fuzzy variance as metric. The adapted version, denoted as partitioning based on fuzzy variance (PFV), is a recursive supervised method, which generates candidate fuzzy partitions, and evaluates these partitions employing fuzzy variance rather than the fuzzy entropy as used in the classification-oriented method.

Let $T R_{f}=\left[\left(x_{1, f}, y_{1}\right), \ldots,\left(x_{N, f}, y_{N}\right)\right]$ be the projection of the training set TR along the input variable $X_{f}$. Let us assume that the values $x_{i, f}$ are sorted in increasing order. Let $I_{f}$ be an interval defined on the universe of $X_{f}$. Let $l_{f}$ and $u_{f}$ be the lower and upper bounds of $I_{f}$. Let $S_{f}$ be the set of values $\left(x_{i, f}, y_{i}\right) \in T R_{f}$ contained in $I_{f}$. Let $P_{I_{f}}=\left\{B_{f, 1}, \ldots, B_{f, K_{P_{I_{f}}}}\right\}$ be a fuzzy partition defined on $I_{f}$, where $K_{P_{I_{f}}}$ is the number of fuzzy sets in $P_{I_{f}}$. Let $S_{f, 1}, \ldots, S_{f, K_{P_{I}}}$ be the subsets of points in $S_{f}$, contained in the support of $B_{f, 1}, \ldots, B_{f, K_{P_{I}}}$, respectively.

The fuzzy mean $\left(\operatorname{FM}\left(P_{I_{f}}\right)\right)$ of a fuzzy partition $P_{I_{f}}$ is defined as the mean of the output values $y_{i}$, corresponding to the instances whose value $x_{i, f} \in S_{f}$, weighted by the membership degrees of $x_{i, f}$ to any $B_{f, j} \in P_{I_{f}}$ :

$$
\operatorname{FM}\left(P_{I_{f}}\right)=\frac{\sum_{j=1}^{K_{P_{I}}} \sum_{\left(x_{i, f}, y_{i}\right) \in S_{f, j}} y_{i} \cdot \mu_{B_{f, i}}\left(x_{j, f}\right)}{\sum_{j=1}^{K_{P_{I}}} \sum_{\left(x_{j, f}, y_{i}\right) \in S_{f, j}} \mu_{B_{f, j}}\left(x_{i, f}\right)}
$$

The fuzzy variance $\left(\operatorname{FVar}\left(P_{I_{f}}\right)\right)$ of a fuzzy partition $P_{I_{f}}$ is defined as:

$$
\operatorname{FVar}\left(P_{I_{f}}\right)=\frac{\sum_{j=1}^{K_{P_{I f}}} \sum_{\left(x_{i f}, y_{i}\right) \in S_{f, j}}\left(y_{i}-\mathrm{FM}\left(P_{I_{f}}\right)\right)^{2} \cdot \mu_{B_{f, j}}^{2}\left(x_{i, f}\right)}{\sum_{j=1}^{K_{I_{f}}} \sum_{\left(x_{i, f}, y_{i}\right) \in S_{f, j}} \mu_{B_{f, j}}^{2}\left(x_{i, f}\right)}
$$

At the beginning of the execution of PFV, $I_{f}$ coincides with the universe of $X_{f}$ and $S_{f}=T R_{f}$. First of all, PFV sorts the values of $T R_{f}$ in ascending order. Afterward, for each value $x_{i, f}$ between $l_{f}$ and $u_{f}$ (at the beginning of the partitioning procedure, $i=1, \ldots, N$ ), PFV defines a strong fuzzy partition $P_{I_{f}}\left(x_{i, f}\right)$ on $I_{f}$ by using three triangular fuzzy sets, namely $B_{f, 1}, B_{f, 2}$ and $B_{f, 3}$, as shown in Fig. 1 . The cores of $B_{f, 1}, B_{f, 2}$ and $B_{f, 3}$ coincide with $l_{f}, x_{i, f}$ and $u_{f}$, respectively. PFV computes $F \operatorname{Var}\left(P_{I_{f}}\left(x_{i, f}\right)\right)$ for each fuzzy partition $P_{I_{f}}\left(x_{i, f}\right)$ induced by each $x_{i, f}$ and selects the optimal value $x_{i, f}^{0}$, which minimizes $F \operatorname{Var}\left(P_{I_{f}}\left(x_{i, f}\right)\right)$.

This value identifies the fuzzy partition $P_{I_{f}}^{0}\left(x_{i, f}^{0}\right)=\left\{B_{f, 1}^{0}, B_{f, 2}^{0}, B_{f, 3}^{0}\right\}$. Let $S_{f, 1}^{0}, S_{f, 2}^{0}$ and $S_{f, 3}^{0}$ be the subsets of points in $S_{f}$, contained in the support of the three fuzzy sets, respectively. Then, PFV applies recursively the procedure for determining the optimal strong fuzzy partition to the intervals $I_{f}=I_{f, 1}^{0}=\left[l_{f}, x_{i, f}^{0}\right]$ and $I_{f}=I_{f, 2}^{0}=\left(x_{i, f}^{0}, u_{f}\right]$ identified by $x_{i, f}^{0}$, by considering $S_{f}=S_{f, 1}^{0}$ and $S_{f}=S_{f, 3}^{0}$, respectively, until the following stopping conditions are met: 


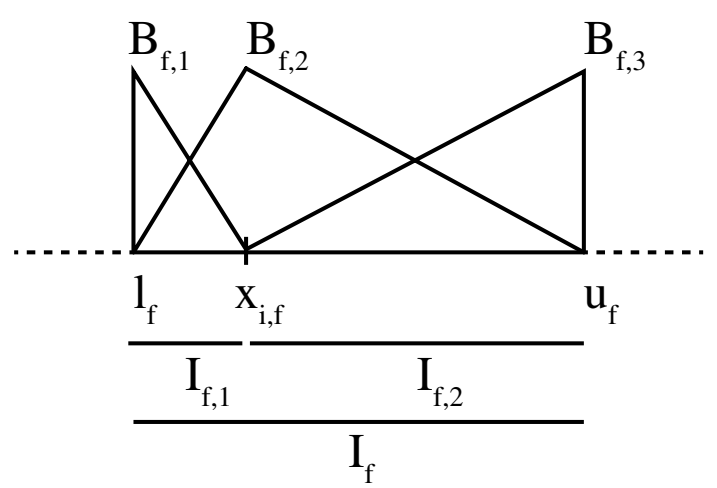

Fig. 1 An example of the fuzzy partition $P_{l_{f}}\left(x_{i, f}\right)$ in the interval $l_{f}$ defined by $l_{f}, x_{i, f}$ and $u_{f}$

- the cardinality $\left|S_{f}\right|$ of $S_{f}$ is lower than a fixed threshold $\lambda_{\text {disc }}$

- the partition fuzzy gain (PFGain $\left(P_{I_{f}}\right)$ ) is lower than a fixed threshold $\epsilon_{\text {disc }}$

PFGain $\left(P_{I_{f}}\right)$ measures the improvement in terms of fuzzy variance of the involved intervals. It is defined as follows:

$$
\begin{aligned}
\operatorname{PFGain}\left(P_{I_{f}}\right)= & F \operatorname{Var}\left(P_{I_{f}}\right)-F \operatorname{Var}\left(P_{I_{f, 1}}\right) \cdot w\left(P_{I_{f, 1}}\right) \\
& -F \operatorname{Var}\left(P_{I_{f, 2}}\right) \cdot w\left(P_{I_{f, 2}}\right)
\end{aligned}
$$

where $w\left(P_{I_{f, s}}\right)$, with $s=1,2$, is defined as:

$$
w\left(P_{I_{f, s}}\right)=\frac{\sum_{g=1}^{K_{P_{f, s}}} \sum_{\left(\mathbf{x}_{i, f}, y_{i}\right) \in S_{f, s}} \mu_{B_{f, g}}^{2}\left(\mathbf{x}_{i, f}\right)}{\sum_{j=1}^{K_{P_{f}}} \sum_{\left(\mathbf{x}_{i, f}, y_{i}\right) \in S_{f}} \mu_{B_{f, j}}^{2}\left(\mathbf{x}_{i, f}\right)}
$$

If no initial partition exists on $I_{f}$ (this occurs when $I_{f}$ coincides with the universe of $X_{f}$ and $S_{f}=T R_{f}$ ), we assume two fuzzy sets $B_{f, 1}$ and $B_{f, 2}$ defined on $I_{f}$, as shown in Fig. 2a. Once the fuzzy partition with three fuzzy sets is accepted, the procedure generates a partition with three fuzzy sets from intervals $I_{f, 1}$ and $I_{f, 2}$ as shown in Fig. 2b. The partitioning of both $I_{f, 1}$ and $I_{f, 2}$ may generate three fuzzy sets in both $\left[l_{f}, x_{i, f}\right]$ and $\left(x_{i, f}, u_{f}\right]$. Actually, the two fuzzy sets, which have the core in $x_{i, f}$ are fused generating a unique fuzzy set. This fusion can be applied at each level of the recursion. The final result is a strong triangular fuzzy partition $P_{f}=\left\{A_{f, 1}, \ldots, A_{f, T_{f}}\right\}$ on $U_{f}$, where $A_{f, j}$ is the $j$-th fuzzy set defined on $U_{f}$.

\section{FRT learning}

Once fuzzy partitions have been defined, the pseudo-code described in Fig. 1 is employed to build the FRT. In the SelectBestFeatureSplits function, we use the expression PFGain $\left(P_{I_{f}}\right)$, defined in Eq. 4 , to determine the best binary split. To calculate the split with the maximum PFGain $\left(P_{I_{f}}\right)$, we evaluate all possible candidates, by grouping together adjacent fuzzy sets into two disjoint groups $Z_{1}$ and $Z_{2}$. The two subsets $G_{1}$ and $G_{2}$ of instances contain the points that belong to the support of the fuzzy sets contained 


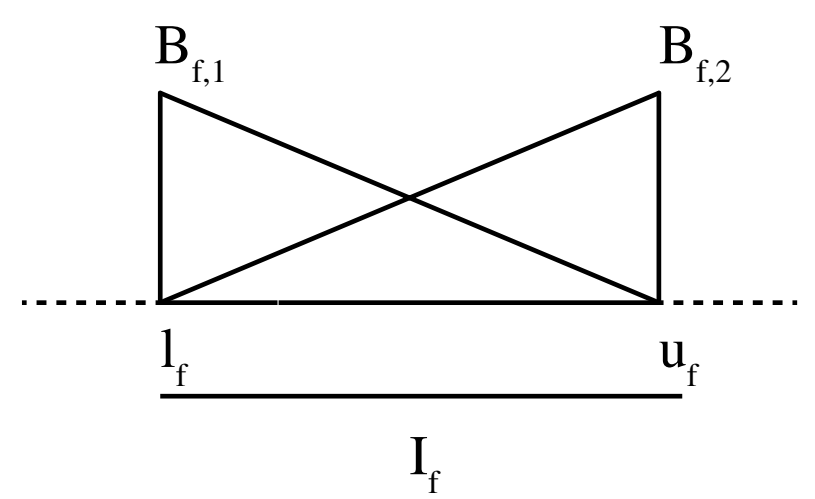

a Initial fuzzy partition defined on $I_{f}$.

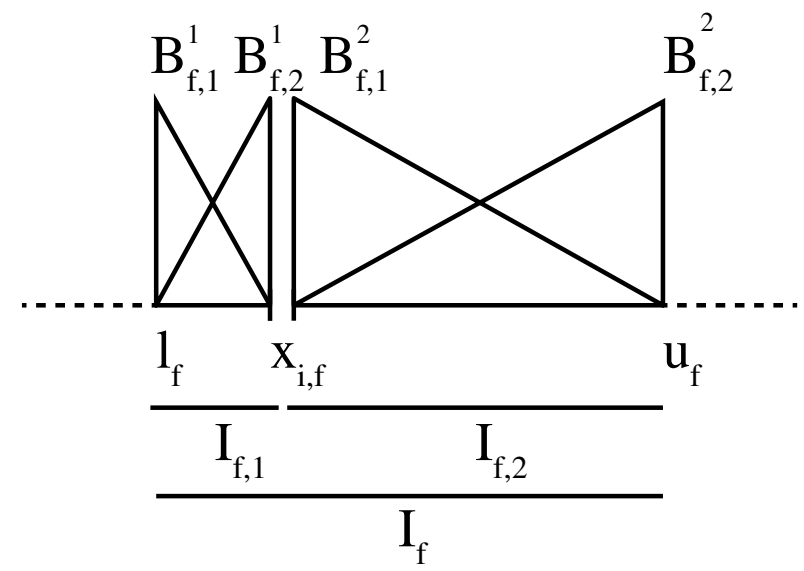

b Accepted fuzzy partition with three fuzzy sets: the fuzzy partitioning procedure continues investigating the possibility of partitioning with three fuzzy sets $I_{f, 1}$ and $I_{f, 2}$.

Fig. 2 An example of the iterative partitioning process

in $Z_{1}$ and $Z_{2}$, respectively. A fuzzy partition with $T_{f}$ fuzzy sets generates $T_{f}-1$ candidates. Starting with $Z_{1}=\left\{A_{f, 1}\right\}$ and $Z_{2}=\left\{A_{f, 2}, \ldots, A_{f, T_{f}}\right\}$, we compute the fuzzy gain by applying Eq. 4, with $P_{f}=\left\{Z_{1}, Z_{2}\right\}$ and cardinality $\left|G_{1}\right|=\sum_{i=1}^{N_{1}} T N\left(\mu_{A_{f, 1}}\left(x_{f, i}\right), \mu_{G}\left(\mathbf{x}_{i}\right)\right)$ and $\left|G_{2}\right|=\sum_{i=1}^{N_{2}} T N\left(\mu_{A_{f, 2}}\left(x_{f, i}\right)+\cdots+\mu_{A_{f, T_{f}}}\left(x_{f, i}\right), \mu_{G}\left(\mathbf{x}_{i}\right)\right)$, where $N_{1}$ and $N_{2}$ are the numbers of instances in the support of the fuzzy sets in $Z_{1}$ and $Z_{2}$, respectively, and $\mu_{G}\left(\mathbf{x}_{i}\right)$ is the membership degree of instance $\mathbf{x}_{i}$ to the parent node. Iteratively, the algorithm investigates all candidates by moving the first fuzzy set in $Z_{2}$ to $Z_{1}$ and computing the corresponding PFGain $\left(P_{I_{f}}\right)$, until $Z_{2}=\left\{A_{f, T_{f}}\right\}$. The pair $\left(Z_{1}, Z_{2}\right)$, which obtains the highest PFGain $\left(P_{I_{f}}\right)$, is used to create the two child nodes. The two nodes contain, respectively, the examples that belong to the support of the fuzzy sets in $Z_{1}$ and $Z_{2}$.

In case of categorical variables the computation cost can become very prohibitive: a categorical variable with $L$ values generates $2^{L-1}-1$ possible combinations. We have adopted the same approach used in [38]: we reduce the possible combinations by sorting the categorical values according to an arbitrary order (by appearance in the dataset). Afterward, we use each categorical value $x_{j, f}$ as splitting point: the first 
subset is composed of all the values $x_{i, f}$ such that $i \leq j$, leaving the rest for the second subset. Therefore, the number of possible partitions is reduced to $L-1$.

The process terminates when one of the following conditions is met (StopMet procedure):

- The number of instances that reach the node is smaller than a fixed threshold $\lambda_{\text {learn. }}$

- The PFGain $\left(P_{I_{f}}\right)$ is lower than a threshold $\epsilon_{\text {learn }}$.

- The tree has reached a maximum fixed depth $\beta$.

The output of the model is computed as in Eq. 1. As we mentioned above, in the case of binary FRTs, an input variable can be considered more than once in the same path from the root to a leaf node, as the partitioning is done in a recursive way where an interval $I_{f}$ can be split into subintervals at different levels. The use of the T-Norm tends to penalize the matching degree of the instance for those input variables that are repeatedly selected along a same path (from the root to a leaf node). To reduce this effect, we use a strategy that remembers each fuzzy set used in the path, and its membership degree is computed and used only the first time it appears; for the subsequent times the membership degree used is 1.

\section{Distributed approach}

We have adapted the distributed implementation proposed in [38] for learning distributed fuzzy decision trees, based on the Map-Reduce paradigm and implemented in Apache Spark. Let $V$ be the number of chunks used for splitting the training set and $Q$ the number of computing units (CUs). Each chunk feds only one Map task, while one CU can process several tasks, both Map and Reduce. Obviously, only $Q$ tasks can be executed in parallel.

The distributed approach follows the same procedure described in the previous sections: first it generates the fuzzy partition of each input variable and then builds the distributed FRT, denoted as DFRT in the following.

With respect to the fuzzy partitioning, we use an approximation of the PFV approach described in "Fuzzy partitioning" section. Indeed, in case of big data, the number of possible candidate splitting points, corresponding to the different values $x_{i, f}$ in the training set for each input variable $X_{f}$, might lead to intractable computation times. To reduce this problem, we limit the number of possible candidate splitting points by adopting an equi-frequency discretization of the domains of each input variable. In particular, as already used in [38], for each input variable $X_{f}$, we sort the values $x_{i f f}$ in the training set and partition the domain of $X_{f}$ into a fixed number $L$ of equi-frequency bins. Then, we aggregate the lists of the bin boundaries generated for each chunk and, for each pair of consecutive bin boundaries, we generate a new bin and compute the required statistics for this bin to be used for computing the fuzzy variance at each iteration of PFV. PFV applies the same process described in "Fuzzy partitioning" section, using the central values of such bins as candidate splitting points. Figure 3 shows the distributed PFV. 


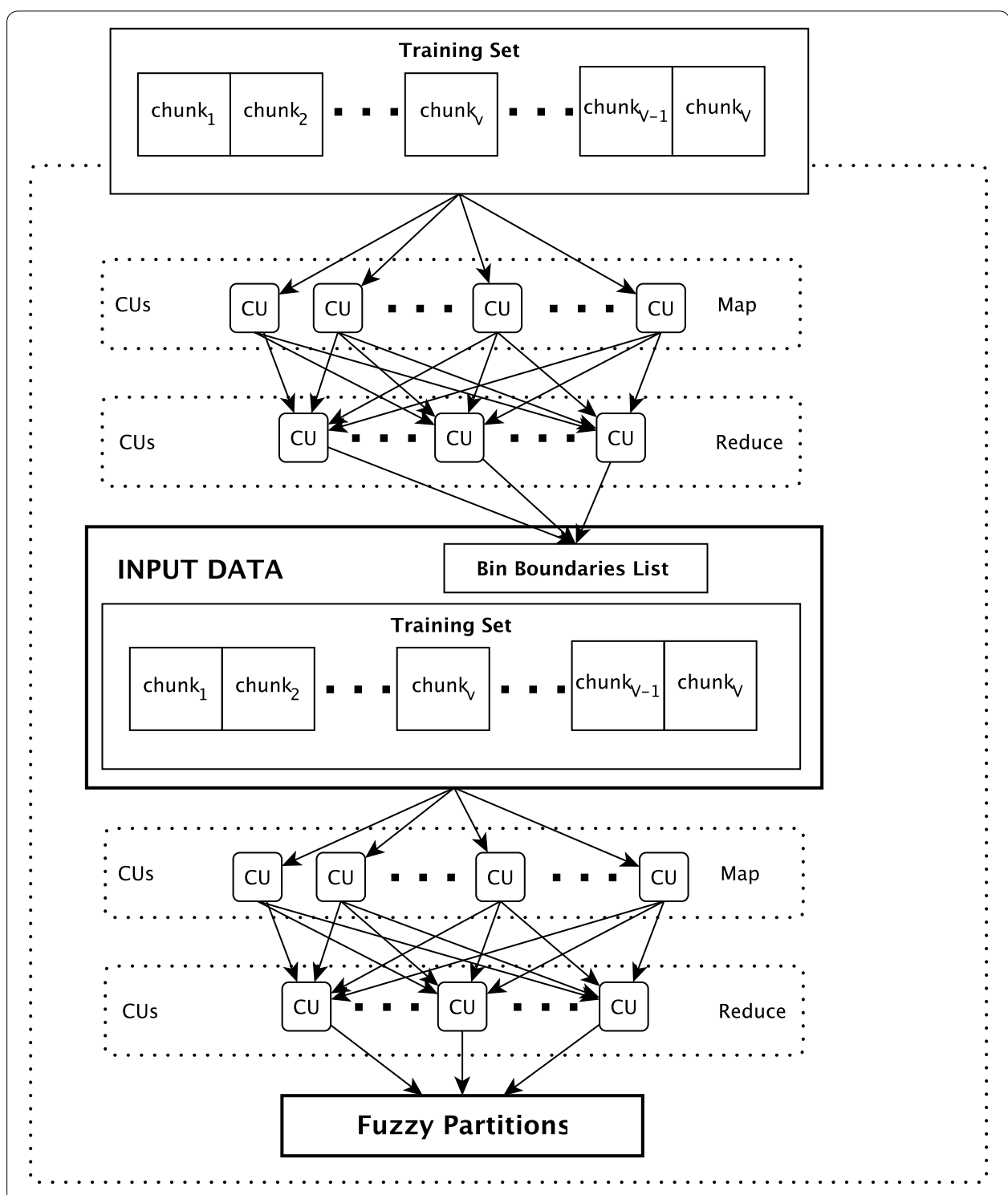

Fig. 3 Distributed partitioning based on fuzzy variance

In the first phase, distributed bin generation, the Map-Reduce implementation of PFV scans the training set to generate at most $V \cdot L$ bin boundaries. Each Map task loads a chunk $v$ of data and, for each continuous input variable $X_{f}$, sorts its values and computes $L$ equi-frequency bin boundaries, including the left and and right extremes of the $X_{f}$ domain in the chunk. Let $B B_{v, f}=\left\{b_{v, f}^{(1)}, \ldots, b_{v, f}^{L}\right\}$ be the sorted list of bin boundaries. The output of the Map task is a key-value pair $\left\langle k e y=f\right.$, value $\left.=B B_{v, f}\right\rangle$, where $f$ is the index of the input variable $X_{f}$ and $B B_{v, f}$ is the sorted list of bin boundaries. Afterwards, each Reduce task is fed by $V$ lists of bin boundaries for the same input variable $X_{f}$, and outputs a key-value pair $\left\langle k e y=f\right.$, value $\left.=B B_{f}\right\rangle$, where $B B_{f}$ is the sorted list of the bin boundaries for that variable $X_{f}$ (obtained by joining all the bin boundaries of each $B B_{v, f}$ ). If the number of bins is equal to the number of values in the interval (one value per bin), it is equivalent to PFV. On the other side, the lower the value of $L$ is, the coarser the 
approximation in determining the fuzzy partition is. We choose $L$ equal to the percentage $\gamma_{\text {disc }}$ of the chunk size.

In the second phase, distributed fuzzy sets generation, we generate the fuzzy partitions for each continuous input variable. Each Map task is fed by the bin boundaries list. Let $x_{i, f} \in S_{f, v}^{(t)}$ be the instances in the chunk $v$ that are contained in the bin $b_{f}^{(t)}$. Then, for each input variable $X_{f}$ and for each bin $b_{f}^{(t)}$ in $B B_{f}$, the Map task computes three statis$\operatorname{tics} \Omega^{\mathbf{t}, \mathbf{v}, \mathbf{f}}=\left\{\Omega_{1}^{t, v, f}, \Omega_{2}^{t, v, f}, \Omega_{3}^{t, v, f}\right\}$, where

- $\Omega_{1}^{t, v, f}$ is the sum of the outputs $y_{i}$ corresponding to $x_{i, f} \in S_{f, v^{j}}^{(t)}$

- $\Omega_{2}^{t, v, f}$ is the sum of the squared outputs $y_{i}^{2}$ corresponding to $x_{i, f} \in S_{f, v^{j}}^{(t)}$

- $\Omega_{3}^{t, v, f}$ is the number of instances $x_{i, f} \in S_{f, v^{\prime}}^{(t)}$

The output of the Map phase is a list of pairs $\left\langle\right.$ key $=f$, value $\left.=\Omega^{\mathbf{t}, \mathbf{v}, \mathbf{f}}\right\rangle$. Each Reduce task is fed by a list of $V$ statistics $\Omega^{\mathbf{t}, \mathbf{v}, \mathbf{f}}$ for the input variable $X_{f}$. In a first phase, the Reduce task performs an element-wise addition for all the three statistics, obtaining $\Omega^{\mathbf{v}, \mathbf{f}}$. Finally, it applies the PFV described in "Fuzzy partitioning" section. Let $\bar{b}_{f}^{(t)}$ be the central point of each bin $b_{f}^{(t)}$. In order to compute efficiently the PFGain $\left(P_{I_{f}}\right)$ given a splitting point $\bar{b}_{f}^{(t)}$, we first compute the following values $G_{f, j}^{(1)}, G_{f, j}^{(2)}, G_{f, j}^{(3)}$ and $G_{f, j}^{(4)}$ by iterating over the bins instead of over the instances, with $f=1 \ldots F$ and $j=1, \ldots, T_{f}$ :

$$
\begin{aligned}
& G_{f, j}^{(1)}=\sum_{b_{f}^{(t)} \in B B_{f}} \mu_{A_{f, j}}\left(\bar{b}_{f}^{(t)}\right) \cdot \Omega_{3}^{t, f} \\
& G_{f, j}^{(2)}=\sum_{b_{f}^{(t)} \in B B_{f}} \mu_{A_{f, j}}^{2}\left(\bar{b}_{f}^{(t)}\right) \cdot \Omega_{3}^{t, f} \\
& G_{f, j}^{(3)}=\sum_{b_{f}^{(t)} \in B B_{f}} \mu_{A_{f, j}}\left(\bar{b}_{f}^{(t)}\right) \cdot \Omega_{1}^{t, f} \\
& G_{f, j}^{(4)}=\sum_{b_{f}^{(t)} \in B B_{f}} \mu_{A_{f, j}}^{2}\left(\bar{b}_{f}^{(t)}\right) \cdot \Omega_{2}^{t, f}
\end{aligned}
$$

Using $G_{f, j}^{(1)}, G_{f, j}^{(2)}, G_{f, j}^{(3)}$ and $G_{f, j}^{(4)}, F M\left(P_{I_{f}}\right)$ can be easily computed as:

$$
F M\left(P_{I_{f}}\right)=\frac{\sum_{j=1}^{K_{I_{f}}} G_{f, j}^{(3)}}{\sum_{j=1}^{K_{P_{f}}} G_{f, j}^{(1)}}
$$


$\operatorname{FVar}\left(P_{I_{f}}\right)$ can be obtained as:

$$
\begin{aligned}
\operatorname{FVar}\left(P_{I_{f}}\right) & =\frac{\sum_{j=1}^{K_{P_{f}}} \sum_{\left(\bar{b}_{f}^{(t)}, y^{(t)}\right) \in S_{f, j}}\left(y^{(t)}-F M\left(P_{I_{f}}\right)\right)^{2} \cdot \mu_{B_{f, j}}^{2}\left(\bar{b}_{f}^{(t)}\right)}{\sum_{j=1}^{K_{P_{f}}} \sum_{\left(\bar{b}_{f}^{(t)}, y^{(t)}\right) \in S_{f, j}} \mu_{B_{f, j}}^{2}\left(\bar{b}_{f}^{(t)}\right)} \\
= & \frac{\sum_{j=1}^{K_{P_{I}}} G_{f, j}^{(4)}+G_{f, j}^{(1)} \cdot F M\left(P_{I_{f}}\right)^{2}-2 \cdot G_{f, j}^{(3)} \cdot F M\left(P_{I_{f}}\right)}{\sum_{j=1}{ }_{P_{I_{f}}} G_{f, j}^{(2)}}
\end{aligned}
$$

Finally, PFGain $\left(P_{I_{f}}\right)$ is computed with Eq. 4. The output of PFV is a fuzzy partition for each input variable. These fuzzy partitions are input to the DFRT learning process.

In DFRT learning, the best split for each node is computed in parallel across the CUs. Figure 4 describes the workflow of the DFRT learning. The DFRT learning is an iterative process, which executes a complete Map-Reduce step at each iteration.

Let $H$ be the number of iterations performed by the algorithm and $h$ be the index of the $h$-th iteration. Let $R$ be the list of nodes to be split, initialized with only one element consisting of the root of the tree. The algorithm iteratively determines the group $R_{h}$ of $|R|$ nodes from $R$. Finally, it performs a Map-Reduce step for distributing the growing process of the tree.

Each Map task, using the corresponding chunk $v$ of data, computes, for each node $N T_{y} \in R_{h}$, a vector $D_{v, y}$ of size $|D|=\sum_{\forall f \in F} T_{f}$ elements. In particular, each element $j$ of $D_{v, y}$ corresponds to the fuzzy set $A_{f, j}$ and its value is equal to $D_{v, y}[j]=\sum_{i=1}^{\left|S_{f, j}\right|} T N\left(\mu_{A_{f, j}}\left(x_{f, i}\right) \cdot \mu_{N T_{y}}\left(\mathbf{x}_{i}\right)\right)$, where $\mu_{N T_{y}}\left(\mathbf{x}_{i}\right)$ is the membership degree of instance $\mathbf{x}_{i}$ to the parent node (for the root of the decision tree, the membership value is equal to 1 ) and the operator $T N$ is a T-norm. The output of these Map tasks are keyvalue pairs $\left\langle k e y=y\right.$, value $\left.=D_{v, y}\right\rangle$ (where $y$ is the index of the node $N T_{y}$ ). Afterward, each Reduce task receives a list of vectors $D_{v, y}$ and creates a vector $D_{y}$ by performing an element-wise addition of all $V$ vectors. Thus, $D_{y}$ stores the cardinality of each input variable from the root to $N T_{y}$ along the overall training set. Then, the Reduce task generates two child nodes and tests the stopping conditions described in "FRT learning" section for both of them. The children generated from each $N T_{y}$ are used to update the tree and $R$ : if a child node is not labeled as leaf, then it is inserted into the list $R$ and employed at the next iterations. The algorithm repeats all the steps until $R$ is empty.

In order to compute the PFGain $\left(P_{I_{f}}\right)$ efficiently (used to test the second stopping criteria), we first compute the values $G_{f, j}^{(1)}, G_{f, j}^{(2)}, G_{f, j}^{(3)}$ and $G_{f, j}^{(4)}$ (Eqs. 6-9 respectively), similar to the distributed PFV algorithm. Further, $\Omega^{\mathbf{l}, \mathbf{f}}$ is already available because it was computed by the distributed PFV algorithm and $\mu_{B_{f, j}}\left(\bar{b}_{f}^{(t)}\right)$ is obtained efficiently by computing the T-Norm using the membership degrees stored in $D_{y}$.

\section{Results and discussion}

In order to test the behavior of the proposed DFRT, we have performed a set of experiments that evaluate the performance in terms of MSE, complexity and scalability related to the size of the datasets and the number of used CUs. MSE is computed as: 


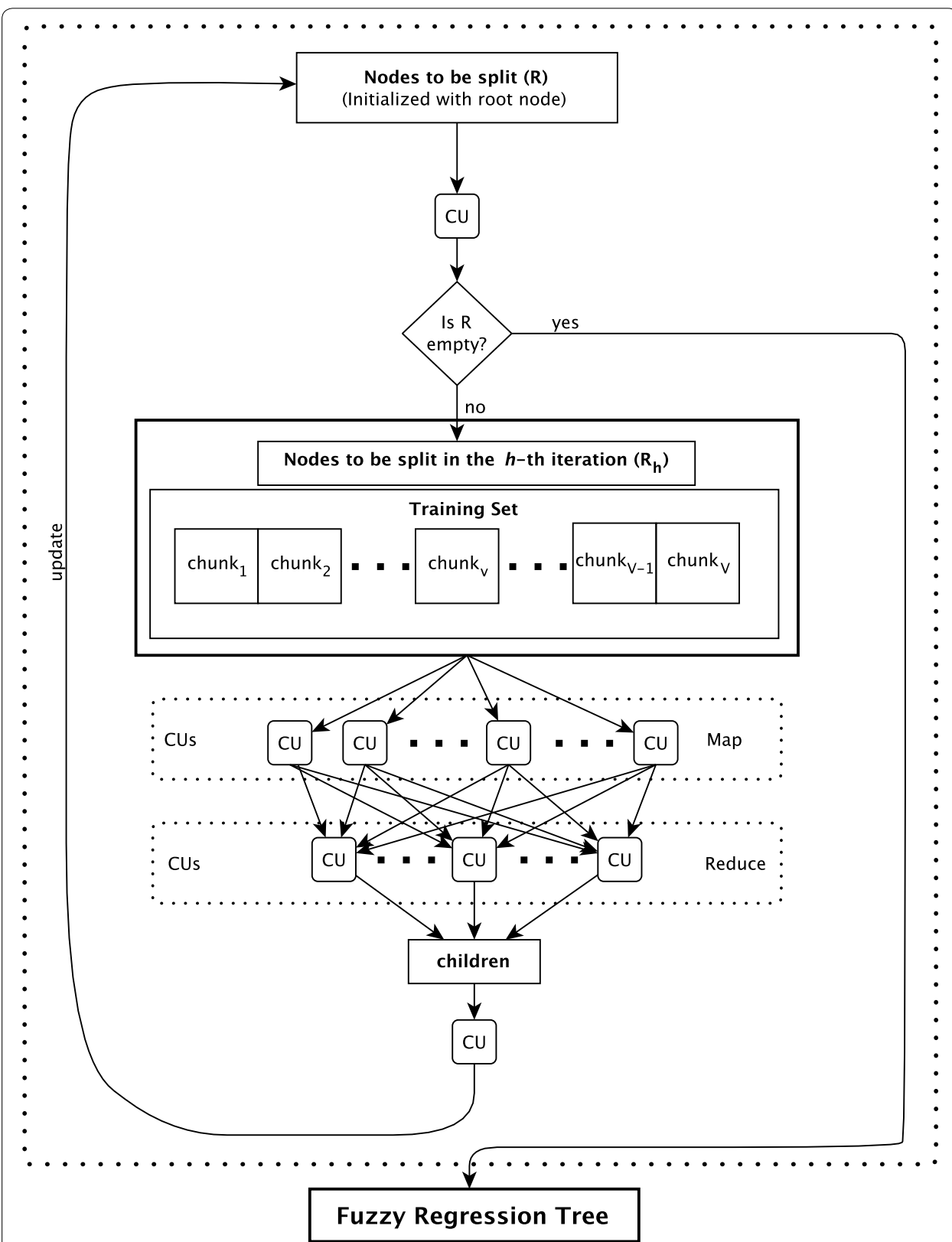

Fig. 4 Distributed fuzzy regression tree learning workflow

$$
\operatorname{MSE}=\frac{\sum_{i=1}^{|D S|}\left(y_{i}-\operatorname{DFRT}\left(\mathbf{x}_{i}\right)\right)^{2}}{|D S|}
$$

where $D S$ stands for each dataset.

Complexity is calculated as the number of nodes of the tree. Scalability is presented by plotting the average execution times versus the number of CUs. 
We have used 8 real world datasets [2]. ${ }^{2}$ These datasets come from the same realworld problem, the Protein Structure Prediction (PSP), which aims to predict the 3D structure of a protein (output variable) based on amino-acid structural continuous variables (inputs). As described in [1], a protein structure (PS) is the three-dimensional arrangement of atoms in a protein molecule. These structures arise because particular sequences of amino-acids in polypeptide chains fold to generate, from linear chains, compact domains with specific 3D structures. The folded domains can serve as modules for building up large assemblies such as virus particles or muscle fibers, or they can provide specific catalytic or binding sites, as found in enzymes or proteins that carry oxygen or regulate the function of DNA. PSP predicts the three-dimensional structures of a protein by using its first structure, its amino-acid sequence, to predict its folding and its secondary, tertiary and quaternary structure $[44,51]$. This makes PSP an essential tool in proteomics since the molecular function of a protein depends on its threedimensional structure, which is often unknown.

The difference among these datasets relies on the number of input variables, varying from 100 (Window2) to 380 (Window9) by steps of 40 input variables. The number of instances is 257560 for all the datasets. To validate the results we have used a ten fold cross-validation (10-CV), being 234638 instances for training and 22922 for test at each iteration.

To the best of our knowledge few real-world big datasets exist for regression problems. Thus, with the aim of enriching the experimental evaluation and extracting more robust conclusions, we have generated 4 synthetic datasets, following the procedure used in [40]: two of them ( $f_{1}$ and $f_{19}$, described in Eqs. 13 and 14) are characterized by 1000 input variables and $10^{5}$ instances, and the remaining two ( $f_{2}$ and $f_{4}$, described in Eqs. 15 and 16$)$ by 100 input variables and $10^{6}$ instances. The generation process consists of generating random instances, where each input variable has a predefined domain, and applying a function over the selected input variables to produce the output value. Let $\mathbf{x}=\left\{x_{1}, x_{2}, \ldots, x_{F}\right\}$ be a random input instance in a predefined domain, $\mathbf{P}$ a random permutation of $\{1,2, \ldots, F\}$ and $M$ an orthogonal matrix of dimension $m \times m$, where $m$ has been fixed to 50 . The definitions of $f_{1}, f_{19}, f_{2}$ and $f_{4}$ are, respetively:

$$
\begin{aligned}
& f_{1}(\mathbf{x})=\sum_{i=1}^{F}\left(10^{6}\right)^{\frac{i-1}{F-1}} \cdot x_{i}^{2} \\
& f_{19}(\mathbf{x})=\sum_{i=1}^{F}\left(\sum_{j=1}^{i} x_{i}\right)^{2} \\
& f_{2}(\mathbf{x})=\sum_{i=1}^{F}\left[x_{i}^{2}-10 \cos \left(2 \pi x_{i}\right)+10\right]
\end{aligned}
$$

\footnotetext{
${ }^{2}$ Obtained from http://ico2s.org/datasets/psp_benchmark.html.
} 
Table 1 Properties of the real-world and synthetic datasets

\begin{tabular}{lccccc}
\hline Dataset & $\boldsymbol{F}$ & $|\boldsymbol{D S}|$ & $\boldsymbol{C}_{\mathbf{1}}(\downarrow)$ & $\boldsymbol{C}_{\mathbf{2}}(\downarrow)$ & $\boldsymbol{C}_{\mathbf{4}}(\boldsymbol{\uparrow})$ \\
\hline Window $_{2}$ & 100 & 257,560 & 0.4973 & 0.1103 & 0.9919 \\
Window $_{3}$ & 140 & 257,560 & 0.4973 & 0.0986 & 0.9918 \\
Window4 $_{4}$ & 180 & 257,560 & 0.4973 & 0.0897 & 0.9917 \\
Window $_{5}$ & 220 & 257,560 & 0.4973 & 0.0805 & 0.9917 \\
Window6 $_{\text {Window7 }}$ & 260 & 257,560 & 0.4973 & 0.0736 & 0.9917 \\
Window $_{8}$ & 300 & 257,560 & 0.4973 & 0.0669 & 0.9917 \\
Window9 $_{f_{1}}$ & 340 & 257,560 & 0.4973 & 0.0613 & 0.9917 \\
$f_{19}$ & 380 & 257,560 & 0.4973 & 0.0571 & 0.9917 \\
$f_{2}$ & 1000 & 100,000 & 0.1614 & 0.0134 & 1.0000 \\
$f_{4}$ & 1000 & 100,000 & 0.0512 & 0.0283 & 1.0000 \\
\hline
\end{tabular}

$$
\begin{aligned}
f_{4}(\mathbf{x})= & f_{1}\left(\left\{x_{P_{1}}, \ldots, x_{P_{m}}\right\} \times M\right) \cdot 10^{6} \\
& +f_{1}\left(\left\{x_{P_{m+1}}, \ldots, x_{P_{F}}\right)\right\}
\end{aligned}
$$

To give a glimpse of the complexity of the problems, we have computed three metrics proposed in [28] for evaluating the correlation between input variables and output. In particular, we have adopted Maximum and Average Feature Correlation to the Output, and Collective Feature Efficiency, denoted as $C_{1}, C_{2}$ and $C_{4}$, respectively, in the original paper. $C_{1}$ and $C_{2}$ measure the maximum and average Spearman correlation values between the input variables and the output (see Eqs. 17 and 18). $C_{4}$ measures the percentage of instances which are difficult to predict (large residual values) with respect to a linear regressor (see Eq. 19).

$$
\begin{aligned}
& C_{1}=\max _{j=1, \ldots, F}\left|\rho\left(x_{j}, y\right)\right| \\
& C_{2}=\sum_{j=1}^{F} \frac{\left|\rho\left(x_{j}, y\right)\right|}{F} \\
& C_{4}=1-\sum_{l} \frac{s_{l}}{|D S|}
\end{aligned}
$$

where $\left(x_{j}, y\right)$ is the projection of the dataset $D S$ along the input variable $X_{j}, \rho\left(x_{j}, y\right)$ is the Spearman correlation between the input variable $X_{j}$ and the output, and $s_{l}$ is the number of instances which have been iteratively marked as linearly classifiable (residual obtained by a linear classifier between the feature $X_{j}$ and $Y$ smaller than 0.01 ), being $X_{j}$ the most correlated feature with respect to $Y$ that has not been yet selected.

The properties for each dataset (number $F$ of input variables, number $|D S|$ of instances, and metrics $C_{1}, C_{2}$ and $C_{4}$ ) are shown in Table 1 . The symbols $\uparrow$ and $\downarrow$ denote that, respectively, high (direct relation) and low (indirect relation) values of the metrics correspond to high complexity. The values highlight that the problems 
Table 2 Parameters for the MLlib DRT and the DFRT

\begin{tabular}{lll}
\hline Parameter & MLlib DRT & DFRT \\
Fuzzy discretization & & $1 \%$ \\
\hline$\lambda_{\text {disc }}$ & - & 0.0001 \\
$\epsilon_{\text {disc }}$ & - & $0.1 \%$ \\
$\gamma_{\text {disc }}$ & - & \\
\hline Regression tree & & - \\
\hline Impurity & Variance & 15 \\
$\beta$ & 15 & 100 \\
$L$ (maximum number of bins) & 100 & $0.5 \%$ \\
$\lambda_{\text {learn }}$ & $0.5 \%$ & 0.0001 \\
$\epsilon_{\text {learn }}$ & 0.0001 & \\
\hline
\end{tabular}

are particularly difficult since there does not exist a high correlation between input variables and output. In particular, the values of $C_{4}$ point out that the output is very difficult to predict by using a linear regressor. In fact, we made some additional experimentation using the distributed linear least squares approach implemented in the Apache Spark MLlib. The results achieved by this algorithm were very poor and thus, for the sake of brevity, we do not present them in this work.

The experiments have been executed on a cluster of computers with one master and six slave nodes equipped with dual Intel Xeon E5-2609v3 $1.90 \mathrm{GHz}$ hexacore processors and 64GB of RAM per node. Each worker node is running the HDFS file system on $4 \times 1$ TB disks and is managed by the 1.5 .2 version of the Apache Spark standalone distribution.

In order to validate the results of our DFRT, we have first compared them to the ones obtained by the distributed regression tree (DRT) of the MLlib Apache Spark's implementation (from version 1.5.2). The parameters used for the execution of DRT and DFRT are shown in Table 2. The comparison is performed in terms of MSE, execution time and scalability. Then, we have compared the results of our DFRT with the ones achieved by the Distributed Random Forests (DRFs) of the MLlib Apache Spark's implementation. Random Forest [4] is an ensemble of regression trees. The effect of using an ensemble is that, asymptotically, the bias of the model is similar to the one obtained by one single tree, while the variance is significantly reduced. As this is one of the key points in fuzzy regression trees, we have compared our DFRT to DRF in terms of variance, in addition to MSE. In "Comparison with Distributed Random Forest” section, we present this comparison and draw some conclusions.

\section{Comparison with DRT}

We will analyze the results for the real and the synthetic datasets separately (first the real-world problems). Tables 3 and 5 show the average training and test MSEs obtained by our DFRT and the MLlib DRT. 
Table 3 Average MSEs obtained by DFRT and DRT on the training and test sets for the realworld problems

\begin{tabular}{llllll}
\hline Problem & \multicolumn{2}{l}{ DFRT } & & & \multicolumn{2}{l}{ DRT } \\
\cline { 2 - 3 } & Training & Test & & Training & Test \\
\hline Window2 & 24.3263 & 24.3978 & 18.1690 & 38.7580 \\
Window3 & 24.2976 & 24.3853 & & 19.0740 & 40.1722 \\
Window4 & 24.2841 & 24.3730 & & 16.1898 & 38.1469 \\
Window5 & 24.2833 & 24.3732 & & 15.9396 & 39.3956 \\
Window6 & 24.2834 & 24.3733 & & 18.2766 & 39.0074 \\
Window7 & 24.2836 & 24.3738 & & 17.4737 & 38.5862 \\
Window8 & 24.2841 & 24.3742 & & 16.2841 & 39.2638 \\
Window9 & 24.2807 & 24.3689 & & 16.9948 & 40.5738 \\
\hline
\end{tabular}

The best results for each dataset have been emphasized in italic font

Table 4 Average complexity of the models obtained by DFRT and DRT for the real-world problems

\begin{tabular}{llllll}
\hline Problem & \multicolumn{2}{l}{ DFRT } & & \multicolumn{2}{l}{ DRT } \\
\cline { 2 - 3 } & \#nodes & \#leaves & & \#nodes & \#leaves \\
\hline Window2 & 666.8 & 333.9 & $36,864.9$ & $18,432.9$ \\
Window3 & 669.6 & 335.3 & $38,560.3$ & $19,280.6$ \\
Window4 & 665.1 & 333.0 & $38,916.0$ & $19,458.5$ \\
Window5 & 665.0 & 333.0 & $39,059.0$ & $19,530.0$ \\
Window6 & 664.9 & 333.0 & 39.9 & $39,224.7$ & $19,575.2$ \\
Window7 & 664.7 & 332.9 & $39,232.9$ & $19,612.8$ \\
Window8 & 664.8 & 333.3 & $39,355.2$ & $19,617.0$ \\
Window9 & 665.5 & & & $19,678.1$ \\
\hline
\end{tabular}

The best results for each dataset have been emphasized in italic font

As we can observe in Table 3, DRT suffers from overfitting. Indeed, the training MSE is much lower than the test MSE. Further, the test MSE obtained by DRT is much higher than the one achieved by DFRT. On the other hand, the training and test MSEs obtained by DFRT are similar, thus showing that DFRT is not prone to overfitting.

Regarding the complexity, Table 4 shows the average number of nodes (inner and leaves) and the average number of leaves. We can observe that the complexity of the models is very similar for the same algorithm for all the real-world datasets (we just observe a slight increase on the numbers of nodes and leaves in the case of DRT). From Table 1 we can observe that the real-world datasets have the same value of the $C_{1}$ metric and the $C_{2}$ metric decreases with the increase of the number of inputs (from Window 2 to Window9). This means that the most correlated input variables belong to the shared 100 input variables (variables from Window 2 ). For this reason, the results do not improve notably by including the rest of the input variables, and therefore are very similar for these datasets. Comparing the algorithms, DRT is much more complex than DFRT in terms of numbers of both nodes and leaves (approximately a difference of one order of magnitude) 
Table 5 Average MSEs obtained by DFRT and DRT on the training and test sets for the synthetic datasets

\begin{tabular}{lllllr}
\hline Problem & \multicolumn{1}{l}{ DFRT } & & \multicolumn{2}{l}{ DRT } \\
\cline { 2 - 3 } & Training & Test & & Training & Test \\
\hline$f_{1}$ & 1.5043 & 1.6654 & 2.3337 & 2.8851 \\
$f_{19}$ & 6.5671 & 7.3154 & & 8.5149 & 12.1510 \\
$f_{2}$ & 3.1378 & 3.2097 & & 3.8151 & 3.8242 \\
$f_{4}$ & 4.2888 & 4.2988 & & 4.5396 & 5.3521 \\
\hline
\end{tabular}

The best results for each dataset have been emphasized in italic font

The results have to be multiplied by $10^{22}, 10^{22}, 10^{5}$ and $10^{33}$ for $f_{1}, f_{19}, f_{2}$ and $f_{4}$ respectively

Table 6 Average complexity of the models obtained by DFRT and DRT for the synthetic datasets

\begin{tabular}{llllll}
\hline Problem & \multicolumn{2}{l}{ DFRT } & & \multicolumn{2}{l}{ DRT } \\
\cline { 2 - 3 } & \#nodes & \#leaves & & \#nodes & \#leaves \\
\hline$f_{1}$ & 634.9 & 317.9 & $43,110.0$ & $21,555.5$ \\
$f_{19}$ & 678.8 & 339.9 & & $37,203.3$ & $18,602.2$ \\
$f_{2}$ & 602.5 & 301.8 & $63,636.6$ & $31,818.8$ \\
$f_{4}$ & 314.4 & 157.7 & $48,858.8$ & $24,429.9$ \\
\hline
\end{tabular}

The best results for each dataset have been emphasized in italic font

In relation to the synthetic datasets, Table 5 shows the results grouped in two blocks: $f_{1}$ and $f_{19}$ (problems with 1000 input variables and $10^{5}$ instances) and $f_{2}$ and $f_{4}$ (problems with 100 input variables and $10^{6}$ instances). We can observe that DFRT outperforms DRT in both training and test sets. Further, we note that DRT suffers from overfitting in $f_{4}$ and more evidently in $f_{19}$. On the other side, DFRT seems not to be affected by this type of problem, thanks to the use of fuzzy rather than crisp boundaries of the decision subspaces. Table 6 shows the average numbers of nodes (again inner and leaves) and leaves. The difference of complexity is more remarkable in this case, being DRT about two orders of magnitude more complex than DFRT.

The analysis of Tables 4 and 6 highlights the capability of the DFRT learning approach to deal with high dimensionality. Indeed, in the DFRTs generated by the learning, a relevant number of input variables are missing, thus proving the input variable selection implicitly performed during the regression tree learning. If, for instance we analyze the synthetic datasets generated from $f_{1}$ and $f_{19}$, we can realize that we have 1000 input variables and, respectively, 634.9 and 678.8 nodes on average. Since the number of nodes also includes the average number of leaves, respectively 317.9 and 339.9 for the two datasets, we have that on average the two DFRTs contain 317 and 338.9 decision nodes. This implies that at most (if a different variable is used in each decision node) 317 and 338.9 out of 1000 input variables are used on average in the DFRT. Thus, we can conclude that a relevant input variable selection is performed, since the number of nodes is lower than the number of input variables. As it occurs for all the regression decision trees, we can conclude that our approach can cope with a high number of input variables by reducing this number during the learning phase.

As regards scalability, we evaluate it with respect to the number of CUs and the dimensionality of the datasets. Figures 5 and 6 show the average execution times (in seconds) 


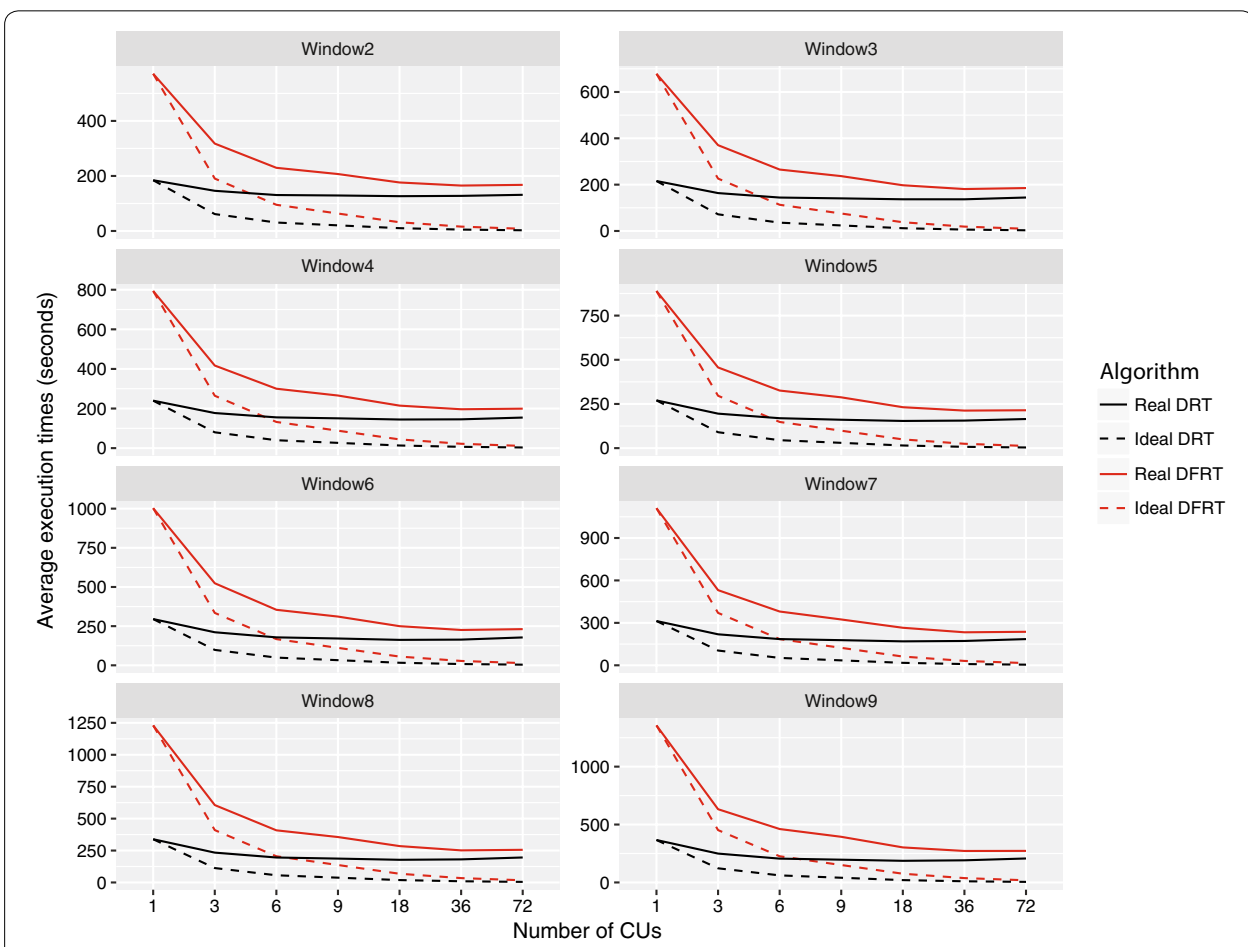

Fig. 5 Average execution times of the DRT and DFRT algorithms for the real-world datasets versus the number of $\mathrm{CUs}$.

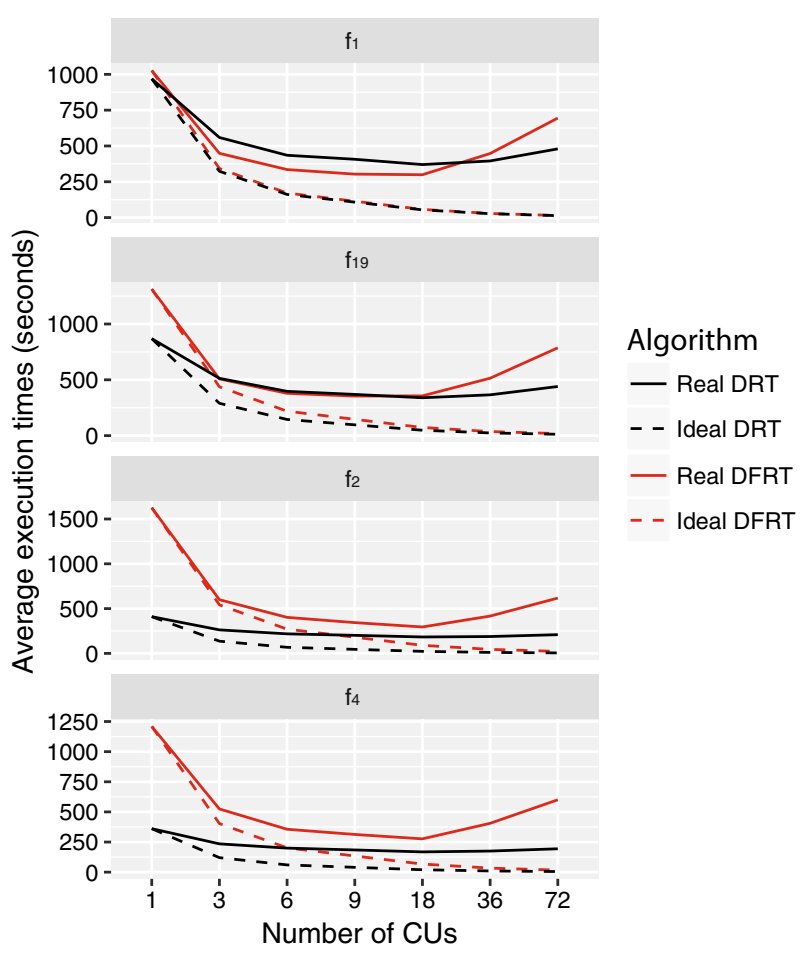

Fig. 6 Average execution times of the DRT and DFRT algorithms for the synthetic datasets versus the number of CUs. 
of DFRT (red lines) and DRT (black lines) for the real world and synthetic datasets, respectively, versus the number of CUs. In the figures, we also show the ideal behavior of the two algorithms (dashed line), whose speedup is linear with respect to the number of CUs.

As we can observe, in the real datasets, DFRT is in general more time consuming, specially when using a small number of CUs. However, as the number of CUs increases, both algorithms need similar computation times.

In the synthetic datasets, we can draw similar conclusions. Furthermore, we notice that, in the case of datasets with a lower number of variables, DRT is much faster than DFRT, specially when using a small number of CUs. In the case of the datasets with a higher number of variables, computation times are similar and, for some number of CUs, DFRT is faster.

There are mainly two aspects which affect the computation time. First, since the tree learning stage consists of an iterative process, the larger the number of nodes of the model, the higher the computation time. Second, the map-reduce phase searches for the best split at each iteration. This is done by analyzing all the possible splits among the candidate nodes. Hence, if the generated model is more complex, a larger number of nodes will be evaluated at each iteration with corresponding increase of the computation time. For high-dimensional datasets, DRT tends to build more complex models (a larger number of nodes) compared to DFRT, thus increasing the computation time. However, for each candidate split point, the computation of the PFGain in DFRT is much more complex than the computation of the corresponding measure in DRT and this increases drastically the computation time of DFRT with respect to DRT. Thus, in the case of datasets with a small number of input variables, DRT is generally faster than DFRT. On the other hand, in the case of datasets with a larger number of input variables, computation times are more similar to each other and sometimes DFRT can outperform DRT (for instance, in the $f_{1}$ dataset).

With respect to the number of CUs, we expect that the larger the number of CUs, the lower the computation time. However, this behavior is affected by the overhead due to communication. We can see how the ratio ideal versus real speedup is directly dependent on the number of CUs: the larger the number of workers, the worse the ratio. This happens because the increase of CUs requires a larger communication among workers and therefore a higher overhead, which is easily observed in the case of the synthetic datasets, where an increase in the number of CUs upon 18 requires a higher computational time than using a lower number of CUs.

However, this behavior is not observed in the case of real-world problems. The reason is that the synthetic datasets have been generated uniformly over the input space, unlike the real-world problems. We observed that, in this scenario, the first phase of the distributed PFV, namely the Distributed Bin Generation, tends to produce a sorted list of bin boundaries per each variable with the maximum number of elements, namely $V \cdot L$. This implies that the second Map-Reduce phase, namely the Distributed Fuzzy Sets Generation, will require a lot of communication to transfer the list of bin boundaries to any worker node for computing the statistics during the execution of the Map task and then to collect the statistics during the execution of the Reduce task. With the increase 
Table 7 Average MSEs and their standard deviation obtained by DFRT and RF on the training and test sets for the real world and synthetic datasets

\begin{tabular}{lrrrrr}
\hline Prob. & \multicolumn{1}{l}{ DFRT } & & \multicolumn{2}{l}{ RF } \\
\cline { 2 - 3 } & Training \pm s.d. & Test \pm s.d. & & Training \pm s.d. & Test \pm s.d. \\
\hline Window2 & $24.3263 \pm 0.18$ & $24.3978 \pm 0.90$ & & $22.9981 \pm 3.02$ & $29.9958 \pm 5.72$ \\
Window3 & $24.2976 \pm 0.18$ & $24.3853 \pm 0.91$ & & $23.1057 \pm 3.43$ & $29.0574 \pm 5.35$ \\
Window4 & $24.2841 \pm 0.18$ & $24.3730 \pm 0.91$ & & $21.3935 \pm 1.31$ & $28.9095 \pm 5.13$ \\
Window5 & $24.2833 \pm 0.18$ & $24.3732 \pm 0.91$ & & $22.1959 \pm 2.85$ & $28.8784 \pm 4.95$ \\
Window6 & $24.2834 \pm 0.18$ & $24.3733 \pm 0.91$ & & $22.5899 \pm 3.53$ & $29.0761 \pm 5.52$ \\
Window7 & $24.2836 \pm 0.18$ & $24.3738 \pm 0.91$ & & $21.9378 \pm 2.56$ & $29.7301 \pm 4.78$ \\
Window8 & $24.2841 \pm 0.18$ & $24.3742 \pm 0.91$ & & $21.0925 \pm 2.64$ & $31.1765 \pm 5.26$ \\
Window9 & $24.2807 \pm 0.18$ & $24.3689 \pm 0.91$ & & $21.8789 \pm 2.82$ & $28.9022 \pm 4.94$ \\
$f_{1}$ & $15.0433 \pm 0.03$ & $16.6537 \pm 0.18$ & & $17.6090 \pm 0.48$ & $15.0783 \pm 0.84$ \\
$f_{19}$ & $6.5671 \pm 0.02$ & $7.3154 \pm 0.11$ & & $6.6059 \pm 0.08$ & $6.8696 \pm 0.09$ \\
$f_{2}$ & $31.3784 \pm 0.02$ & $32.0971 \pm 0.14$ & & $32.2631 \pm 0.28$ & $29.1347 \pm 0.75$ \\
$f_{4}$ & $428.8752 \pm 0.47$ & $429.8814 \pm 3.39$ & & $426.1867 \pm 0.74$ & $427.2893 \pm 3.40$ \\
\hline
\end{tabular}

The best results for each dataset have been emphasized in italic font

The results have to be multiplied by $10^{21}, 10^{22}, 10^{4}$ and $10^{31}$ for $f_{1}, f_{19}, f_{2}$ and $f_{4}$ respectively

of the number of nodes, the overhead due to this communication grows and makes the advantage of having a higher number of CUs ineffective.

\section{Comparison with Distributed Random Forest}

In this section, we aim to compare the results obtained by DFRT with one of the stateof-the-art algorithms available for regression problems with big data, namely DRF. We adopt DRF for two reasons. First, DRF is available in the Spark MLlib library. Second, it has been proved that, comparing an RT versus an RF (ensemble of RTs) [41], the bias of $\mathrm{RF}$ is asymptotically similar but the variance is reduced. Thus, we will compare DFRT with DRF with respect to variance, in addition to MSE (we will use the standard deviation to improve the readability of the results).

We execute DRF by using the default values recommended by the authors. Furthermore, we have set the two parameters, which do not have default values, namely the maximum depth for any tree and the number of trees, which compose the ensemble, to 10 (parameter $\beta$ ) and 20, respectively. In a preliminary experimentation, we tried different (and larger) values for these parameters. However, we could not run the algorithm on a single $\mathrm{CU}$ because of memory overflow. In order to compare the algorithms under similar environments, we finally chose the previous parametrization.

Table 7 shows the average training and test MSEs with their respective standard deviations. We can observe that DFRT achieves a lower test MSE than DRF in the real world problems, while DRF obtains a lower test MSE than DFRT in the synthetic datasets. In the real datasets, we observe that RF is affected by overfitting (the test MSE is larger than almost $30 \%$ of the training MSE). However, in the case of synthectic datasets, training and test MSEs are very similar for DFRT and RF. We have applied a Wilcoxon Signed Rank Test [46] to evaluate whether there exists a statistical difference between the two algorithms. We adopted $\alpha=0.05$ as confidence level and obtained $\mathrm{p}$-value $=0.1697$, which points out that there is no statistical evidence that these algorithms are different. 
As regards the standard deviation, DFRTs are characterized by smaller standard deviations than DRFs, except for $f_{19}$. Moreover, the standard deviation obtained by DFRT is small (always less than a 10\% of the MSE), while in the case of RF it is greater, up to $20 \%$ in the case of real world problems. Again, we have applied a Wilcoxon Signed Rank Test with $\alpha=0.05$. We have obtained $\mathrm{p}$-value $=0.0046$, thus pointing out that the standard deviation obtained by DFRT is statistically lower than the one achieved by DRFs. This result is very interesting, considering the observation made in [41] regarding the variance of the MSEs obtained by RFs.

\section{Conclusions}

In this work we have proposed a distributed fuzzy regression tree learning algorithm (DFRT), following the Map Reduce programming model to cope with Big Data problems. DFRT uses a novel distributed fuzzy discretizer (PFV), adapted from [38] to deal with regression problems. PFV generates a strong fuzzy partition based on the fuzzy variance. Then, DFRT applies an iterative process to generate a binary fuzzy regression tree. The distributed version of the algorithm has been implemented using the Apache Spark framework.

We have used 8 real-world datasets, generated from the same problem. In particular, all of them have the same number of instances, but they differ in the number of input variables. In addition, we have generated 4 synthetic datasets: two of them with 1000 input variables and $10^{5}$ instances, and the others with 100 input variables and $10^{6}$ instances. We have compared the results obtained by our approach with the distributed regression tree (DRT) and the Distributed Random Forests (DRFs) implemented in the MLlib Apache Library (included in the 1.5.2 version of the Apache Spark).

Results show that DFRT scales similar to the DRT algorithm, but the results obtained by DFRT outperform in all the cases the DRT in terms of MSE on the test set and also in terms of complexity (number of nodes and leaves). In terms of execution time, even if the scalability behavior is similar to DRT, the DFRT algorithm is slower. We have observed that, in the case of the smaller datasets (less number of features), DRT is generally faster than DFRT. On the other hand, DFRT is faster (or at least similar) than DRT for the case of datasets with a large number of input variables.

As regards the comparison with DRF, the results show that DFRT and DRF are not statistically different in terms of test MSE, but DFRT outperforms DRF in terms of standard deviation.

\section{Abbreviations}

CV: cross-validation; DT: decision tree; DFRT: distributed fuzzy regression tree; DRF: Distributed Random Forest; DRT: distributed regression tree; FRT: fuzzy regression tree; HDFS: Hadoop Distributed FileSystem; MR: Map Reduce; MSE: mean squared error; PFV: partitioning based on fuzzy variance; RF: Random Forest; RT: regression tree.

\section{Authors' contributions}

Authors propose a distributed fuzzy regression tree learning algorithm following the Map Reduce programming model to cope with Big Data problems. Authors adapt a novel distributed fuzzy discretizer (PFV) from [38] to deal with regression problems. The algorithm has been implemented using the 1.5.2 version of Apache Spark framework. All authors read and approved the final manuscript.

\section{Author details}

${ }^{1}$ Departamento de Sistemas Informáticos/I3A, Universidad de Castilla-La Mancha, 02071 Albacete, Spain. ${ }^{2}$ Dipartimento di Ingegneria dell'Informazione, Largo Lucio Lazzarino 1, 56122 Pisa, Italy. 


\section{Acknowlegements}

The authors would like to acknowledge Dr. Armando Segatori for the precious suggestions and explanations on the implementation of the distributed fuzzy decision trees for big data classification problems.

\section{Competing interests}

The authors declare that they have no competing interests.

\section{Availability of data and materials}

Real world datasets can be downloaded from the website $h$ ttp://ico2s.org/datasets/psp_benchmark.html. To download the synthetic datasets, please contact the corresponding author Javier Cózar javier.cozar@uclm.es.

\section{Funding}

This work has been partially supported by the project PRA 2017"IoT e Big Data: metodologie e tecnologie per la raccolta e l'elaborazione di grosse moli di dati", funded by the University of Pisa. This work has been partially funded by the Spanish Research Agency (AEI/MINECO) and FEDER (UE) through projects TIN2013-46638-C3-3-P and TIN2016-77902-C3-1-P. This work has been partially funded by the Junta de Comunidades de Castilla-La Mancha and FEDER (UE) funds through project SBPLY/17/180501/000493. Javier Cózar has also been funded by the MICINN grant FPU12/05102.

\section{Publisher's Note}

Springer Nature remains neutral with regard to jurisdictional claims in published maps and institutional affiliations.

Received: 22 August 2018 Accepted: 29 November 2018

Published online: 12 December 2018

\section{References}

1. Arana-Daniel N, Gallegos AA, López-Franco C, Alanís AY, Morales J, López-Franco A. Support vector machines trained with evolutionary algorithms employing kernel adatron for large scale classification of protein structures. Evol Bioinform. 2016;12:285-302.

2. Bacardit J, Krasnogor N. The icos psp benchmarks repository; 2008. http://ico2s.org/datasets/psp_benchmark.html. Accessed 3 Dec 2018.

3. Berzal F, Cubero JC, Marın N, Sánchez D. Building multi-way decision trees with numerical attributes. Inf Sci. 2004;165(1):73-90.

4. Breiman L. Random forests. Mach Learn. 2001:45(1):5-32.

5. Breiman L, Friedman JH, Olshen RA, Stone CJ. Classification and regression trees. Monterey: Wadsworth \& Brooks; 1984.

6. Cheng HD, Chen JR. Automatically determine the membership function based on the maximum entropy principle. Inf Sci. 1997;96(3-4):163-82.

7. Cózar J, delaOssa L, Gámez JA. Learning tsk-0 linguistic fuzzy rules by means of local search algorithms. Appl Soft Comput. 2014;21:57-71

8. Cózar J, delaOssa L, Gámez JA. Tsk-0 fuzzy rule-based systems for high-dimensional problems using the apriori principle for rule generation. In: Rough sets and current trends in computing, lecture notes in computer science, vol 8536. New York: Springer International Publishing: 2014. p. 270-9.

9. Dean J, Ghemawat S. Mapreduce: simplified data processing on large clusters. Commun ACM. 2008;51(1):107-13.

10. Slowiński R. Fuzzy sets in decision analysis, operations research and statistics, vol 1. US: Springer; 2012.

11. Diao R, Sun K, Vittal V, O'Keefe RJ, Richardson MR, Bhatt N, Stradford D, Sarawgi SK. Decision tree-based online voltage security assessment using pmu measurements. IEEE Trans Power Syst. 2009;24(2):832-9.

12. Dubois D, Prade H. Unfair coins and necessity measures: towards a possibilistic interpretation of histograms. Fuzzy Sets and Systems. 1983;10(1-3):15-20.

13. Fonarow GC, Adams KF, Abraham WT, Yancy CW, Boscardin WJ, Committee ASA. Risk stratification for inhospital mortality in acutely decompensated heart failure: classification and regression tree analysis. JAMA. 2005:293(5):572-80.

14. Franklin J. The elements of statistical learning: data mining, inference and prediction. Math Intell. 2005;27(2):83-5.

15. Garcia S, Luengo J, Sáez JA, Lopez V, Herrera F. A survey of discretization techniques: taxonomy and empirical analysis in supervised learning. IEEE Trans Knowl Data Eng. 2013;25(4):734-50.

16. Goetz T. The decision tree: taking control of your health in the new era of personalized medicine. Emmaus: Rodale: 2010.

17. Gupta A, Mehrotra KG, Mohan C. A clustering-based discretization for supervised learning. Stat Probab Lett. 2010;80(9):816-24.

18. Haskell RE. Regression tree fuzzy systems. In: Proceedings of the ICSC symposium on soft computing, fuzzy logic, artificial neural networks and genetic algorithms, University of Reading, Whiteknights, Reading, England; 1996. p. 26-8.

19. Hastie T, Tibshirani R, Friedman J. The elements of statistical learning: data mining, inference, and prediction. Springer series in statistics, vol 10, 1st ed. New York: Springer; 2001.

20. Izrailev S, Agrafiotis D. A novel method for building regression tree models for qsar based on artificial ant colony systems. J Chem Inf Comput Sci. 2001;41(1):176-80.

21. Keller JM, Gray MR, Givens JA. A fuzzy k-nearest neighbor algorithm. IEEE Trans Syst Man Cybern. 1985:4:580-5.

22. Kim H, Loh WY. Classification trees with unbiased multiway splits. J Am Stat Assoc. 2001;96(454):589-604.

23. Kotsiantis S, Kanellopoulos D. Discretization techniques: a recent survey. GESTS Int Trans Comput Sci Eng. 2006;32(1):47-58. 
24. Leathwick J, Elith J, Francis M, Hastie T, Taylor P. Variation in demersal fish species richness in the oceans surrounding new zealand: an analysis using boosted regression trees. Mar Ecol Prog Ser. 2006;321:267-81.

25. Leskovec J, Rajaraman A, Ullman JD. Mining of massive datasets. Cambridge: Cambridge University Press; 2014.

26. Leskovec J, Rajaraman A, Ullman JD. Mining of massive datasets. Cambridge: Cambridge university press; 2014.

27. Liu H, Hussain F, Tan CL, Dash M. Discretization: an enabling technique. Data Mining Knowl Discov. 2002;6(4):393-423.

28. Maciel Al, Costa IG, Lorena AC Measuring the complexity of regression problems. In: 2016 international joint conference on neural networks (IJCNN). New York: IEEE; 2016. p. 1450-7.

29. Medasani S, Kim J, Krishnapuram R. An overview of membership function generation techniques for pattern recognition. Int J Approx Reason. 1998;19(3-4):391-417.

30. Meng X. Mllib: Scalable machine learning on spark. In: Spark Workshop April; 2014.

31. Mori H, Kosemura N, Ishiguro K, Kondo T. Short-term load forecasting with fuzzy regression tree in power systems. In: 2001 IEEE international conference on systems, man, and cybernetics, vol 3. New York: IEEE; 2001. p. 1948-53.

32. Nieradka G, Butkiewicz B. A method for automatic membership function estimation based on fuzzy measures. In: International fuzzy systems association world congress. Berlin: Springer; 2007. p. 451-60

33. Olaru C, Wehenkel L. A complete fuzzy decision tree technique. Fuzzy Sets Syst. 2003;138(2):221-54

34. Pedrycz W. Why triangular membership functions? Fuzzy Sets Syst. 1994;64(1):21-30.

35. Prasad AM, Iverson LR, Liaw A. Newer classification and regression tree techniques: bagging and random forests for ecological prediction. Ecosystems. 2006;9(2):181-99.

36. Quinlan RJ. Learning with continuous classes. In: 5th Australian joint conference on artificial intelligence. Singapore: World Scientific; 1992. p. 343-8.

37. Segal MR. Regression trees for censored data. Biometrics. 1988:44:35-47.

38. Segatori A, Marcelloni F, Pedrycz W. On distributed fuzzy decision trees for big data. IEEE Trans Fuzzy Syst. 2018;26(1):174-92.

39. Suárez A, Lutsko JF. Globally optimal fuzzy decision trees for classification and regression. IEEE Trans Pattern Anal Mach Intell. 1999;21(12):1297-311.

40. Tang K, Li X, Suganthan PN, Yang Z, Weise T. Benchmark functions for the cec2010 special session and competition on large-scale global optimization. Tech. rep. nature inspired computation and applications laboratory; 2009.

41. Wager S Asymptotic theory for random forests. arXiv preprint; 2014. arXiv:14050352.

42. Ward JS, Barker A. Undefined by data: a survey of big data definitions. arXiv preprint; 2013. arXiv:13095821.

43. Weber R. Fuzzy-id3: a class of methods for automatic knowledge acquisition. In: Proceedings of the 2nd international conference on fuzzy logic and neural networks; 1992.

44. Westhead DR, Thornton JM. Protein structure prediction. Curr Opin Biotechnol. 1998;9(4):383-9.

45. White T. Hadoop: the definitive guide. Sebastopol: O'Reilly Media, Inc.; 2012.

46. Wilcoxon F. Individual comparisons by ranking methods. Biometrics Bull. 1945;1 (6):80-3.

47. Yuan Y, Shaw MJ. Induction of fuzzy decision trees. Fuzzy Sets Syst. 1995;69(2):125-39.

48. Zaharia M. Apache Spark MLlib; 2009. http://spark.apache.org/mllib/. Accessed 26 Sept 2017.

49. Zaharia M, Chowdhury M, Franklin MJ, Shenker S, Stoica I. Spark: cluster computing with working sets. HotCloud. 2010;10(10-10):95.

50. Zeinalkhani M, Eftekhari M. Fuzzy partitioning of continuous attributes through discretization methods to construct fuzzy decision tree classifiers. Inf Sci. 2014;278:715-35.

51. Zhang Y, Skolnick J. The protein structure prediction problem could be solved using the current pdb library. Proc Natl Acad Sci. 2005;102(4):1029-34.

52. Zheng $Y$, Liu L, Wang $L$, Xie X. Learning transportation mode from raw gps data for geographic applications on the web. In: Proceedings of the 17th international conference on World Wide Web, New York: ACM; 2008. p. 247-56.

\section{Submit your manuscript to a SpringerOpen ${ }^{\circ}$ journal and benefit from:}

- Convenient online submission

- Rigorous peer review

- Open access: articles freely available online

High visibility within the field

Retaining the copyright to your article

Submit your next manuscript at $\gg$ springeropen.com 